\title{
BMJ open The COMPlaints After Stroke (COMPAS) study: protocol for a Dutch cohort study on poststroke subjective cognitive complaints
}

\author{
Marielle W A van Rijsbergen, ${ }^{1}$ Ruth E Mark, ${ }^{1}$ Paul L M de Kort, ${ }^{2}$ \\ Margriet M Sitskoorn ${ }^{1}$
}

\section{To cite: van}

Rijsbergen MWA, Mark RE, de Kort PLM, et al. The COMPlaints After Stroke (COMPAS) study: protocol for a Dutch cohort study on poststroke subjective cognitive complaints. BMJ Open 2013;3:e003599. doi:10.1136/bmjopen-2013003599

- Prepublication history for this paper is available online. To view these files please visit the journal online (http://dx.doi.org/10.1136/ bmjopen-2013-003599).

Received 15 July 2013 Accepted 9 August 2013

For numbered affiliations see end of article.

\footnotetext{
Correspondence to

Dr Ruth E Mark;

r.e.mark@tilburguniversity. edu
}

\section{ABSTRACT}

Background: Although many studies have assessed poststroke objective cognitive impairment, only a few have evaluated patients' subjective cognitive complaints (SCC). Although these SCC are found to be common in the early and chronic phases after stroke, knowledge about their risk factors, course over time, differences with healthy controls and their diagnostic relevance is limited. The aim of the COMPlaints After Stroke (COMPAS) study is therefore to determine the possible risk factors, prognosis, time course and predictive value of SCC in the first 2 years after stroke.

Methods and design: A prospective cohort study is conducted in which patients are compared to non-stroke controls at 3, 6, 12 and 24 months after stroke. Approximately 300 patients are recruited from the stroke units of three hospitals in the Netherlands, while 300 controls are sought among the relatives (spouses excluded) and social networks of participants. A wide range of subjective and objective variables is assessed in both groups using interviews, questionnaires and neuropsychological assessment. The primary outcomes include SCC and objective cognitive impairment, whereas secondary outcomes are quality of life, subjective recovery and daily life functioning.

Ethics and dissemination: The study is being carried out in agreement with the Declaration of Helsinki and the Medical Research Involving Human Subjects Act. The protocol has been approved by the medical ethics committees of the participating centres and all participants give written informed consent. The results will be published in peer-reviewed journals and disseminated to the medical society and general public.

Discussion: The COMPAS study is the first to systematically evaluate poststroke SCC in a prospective longitudinal design, taking a wide range of subjective and objective variables into account. The results obtained can be used to accurately inform patients and their families, as well as to develop patient-tailored intervention programmes to ultimately improve stroke patient care.

\section{BACKGROUND}

Poststroke cognitive impairment is common after stroke and can be evaluated either

\section{ARTICLE SUMMARY}

Strengths and limitations of this study

- Strengths of this study are that it is the first prospective cohort study on subjective cognitive complaints in patients with stroke in which patients and controls are evaluated at multiple assessments, while at the same time a wide range of subjectively and objectively measured variables are taken into account.

- A limitation is that the most seriously affected patients are unable to participate in the study. This may reduce the generalisability of the results to the stroke population as a whole.

objectively, using neuropsychological tests (ie, objective cognitive performances (OCP)), or subjectively, using interviews or self-report questionnaires (ie, subjective cognitive complaints (SCC)). Until now, the majority of studies on poststroke cognitive sequelae have focused on OCP without also evaluating patients' SCC. However, individuals' performances in test situations do not always correspond to those in daily life and vice versa. ${ }^{12}$ Evaluating one can therefore not be used to draw conclusions about the other.

In a recent systematic review, we found that SCC are common in the early and chronic phases after stroke and that they tend to increase over time. ${ }^{3}$ The prevalence rates vary between $28.6 \%$ and $92.0 \%$ and complaints about memory, mental speed and concentration were found to be those most commonly reported. ${ }^{3}$ Furthermore, patients and their proxies showed moderate agreement on the prevalence and severity of patients' SCC. ${ }^{3}$ However, one of the main problems in most of these studies is that there is no 'gold standard' to define and measure SCC, resulting in heterogenic findings. In our review, we suggested that it is important to differentiate between content of 
SCC (SCCc) and worrying about SCC (SCCw), as these are two different concepts. ${ }^{3}$ The first focuses on the specific cognitive difficulties respondents say they experience, while the second indicates whether participants find them worrisome, irritating and whether they say they hinder daily life. A few studies have made this distinction so far. ${ }^{2}{ }^{4}$ However, the majority of research on poststroke SCC has evaluated SSCc and not SSCw, probably without being aware of the difference between these aspects of SCG. ${ }^{3}$

Furthermore, we found in our review that poststroke SCG are inconsistently associated with demographic and clinical characteristics, current depressive symptoms and $\mathrm{OCP}^{3}$ but they may predict future emotional and cognitive decline. ${ }^{5}{ }^{6}$ However, most of the research on SCC after stroke carried out so far is limited in that: unvalidated methods for assessing SCC were used, there was no nonstroke control group included, and the focus was on a specific subsample of stroke patients (eg, home-living patients only), thereby impairing the generalisability of the results. While SCC are common among stroke patients, knowledge about the following aspects is only limited or practically non-existent: the risk profile for developing SCC; their course over time; their impact on quality of life (QOL), subjective recovery and activities in daily life (ADL) functioning; and their prognostic implications.

In the general non-stroke population, however, SCC have been more frequently evaluated, in particular memory-related SCC reported by the elderly. ${ }^{78}$ Factors found to be associated with these complaints include: demographic characteristics (higher age, women, lower education), psychological distress, somatic symptoms, personality traits (neuroticism in particular) and vascular risk factors. ${ }^{7-11}$ Furthermore, they are thought to be clinically relevant in this group because of their association with increased healthcare consumption, reduced QoL, current OCP (this link is not always found) and their predictive value for future cognitive decline. ${ }^{7} 812$ Whether this also applies to poststroke SCC is unknown. More systematic research is therefore needed to gain further knowledge about SCC among stroke survivors, to be able to accurately inform patients and their relatives, to develop adequate treatment programmes and to ultimately improve poststroke care.

We therefore designed the COMPlaints After Stroke (COMPAS) study in which we have four main aims:

- Determine the prevalence, profile and course over time of SCCc and SCCw.

- Identify the risk profile for reporting SCC.

- Evaluate their predictive value for future cognitive functioning.

- Determine the effect of SCG on QoL, subjective recovery and ADL functioning.

Here we describe the design and protocol of the COMPAS study, which is the first prospective cohort study of SCC in patients with stroke, evaluating both patients and controls, while at the same time a wide range of variables is taken into account.

\section{METHODS AND ANALYSIS}

\section{Design}

A multicentre, prospective cohort study of patients with stroke and controls is performed. We started in 2009 and the final measurements will be made in 2014 . Patients are evaluated five times, starting at the clinical phase (T0), followed by an assessment at 3 months (T1), 6 months (T2), 1 year (T3) and 2 years (T4) after stroke. Controls are seen at the same time intervals, starting at $\mathrm{T} 1$.

\section{Study population}

Patients with stroke are recruited consecutively from the stroke units of three hospitals in the Netherlands, including the St Elisabeth and TweeSteden Hospitals in Tilburg and the Maxima Medical Centre in Veldhoven. The control group consists of a sample from the nonstroke general population and is recruited among the relatives and the social networks of participants in the COMPAS study. Spouses of patients with stroke are excluded from the control group since they are at a higher risk of having physical, cognitive and psychosocial problems themselves due to the fact that their partner has suffered a stroke. ${ }^{13} 14$

\section{Inclusion criteria}

- Clinical diagnosis of a first or recurrent ischaemic or haemorrhagic stroke (for patients only).

- At least 18 years old (no upper age limit). Exclusion criteria

- Pre-existent health problems interfering with cognitive functioning, including for example:

- Cognitive decline (as defined by a score $>3.6$ on the short version of the Informant Questionnaire on Cognitive Decline in the Elderly; IQCODE).$^{15}$

- A recent history of severe psychopathology (eg, suicide attempts, alcohol- or drug misuser, diagnosed personality or mood disorders).

- Severe physical comorbidity (eg, malignant diseases, progressive neurological conditions).

- Severe communication difficulties (eg, insufficient understanding of the Dutch language, severe aphasia, blindness or deafness).

\section{Procedure}

Eligible patients receive oral and written information about the study from their treating physician during the clinical phase (T0). Demographic and clinical characteristics are documented and patients are scheduled for the first assessment 3 months after stroke (T1), during which written informed consent is obtained for inclusion to be definite. Participants acknowledge that they have the intention to complete all four assessments, and that they are allowed to end their participation at any time. For the follow-up assessments (T2-T4), patients are informed by letter and telephone and invited to participate after which an appointment is scheduled.

Potential controls receive oral and written information about the study from the researcher after which they are 
Table 1 Primary and secondary outcomes in the COMPAS study

\begin{tabular}{|c|c|c|c|c|c|c|}
\hline & Instrument & TO & T1 & T2 & T3 & T4 \\
\hline \multicolumn{7}{|l|}{ Primary outcomes } \\
\hline \multirow[t]{2}{*}{ SCC } & Cognitive Failures Questionnaire ${ }^{1617}$ & & $X$ & $X$ & $\mathrm{X}$ & $x$ \\
\hline & Checklist for Cognitive and Emotional Consequences ${ }^{5}$ & & $\mathrm{X}$ & $\mathrm{X}$ & $\mathrm{X}$ & $\mathrm{X}$ \\
\hline \multicolumn{7}{|c|}{ ( } \\
\hline Global cognitive functioning & Mini-Mental State Examination & & $\mathrm{X}$ & & $X$ & $\mathrm{X}$ \\
\hline $\begin{array}{l}\text { Visual perception and } \\
\text { construction }\end{array}$ & Rey Complex Figure Test—copy trial & & $\mathrm{X}$ & & $\mathrm{X}$ & $X$ \\
\hline \multirow[t]{2}{*}{ Mental speed/attention } & Stroop Colour word test-cards 1 and 2 & & $X$ & & $\mathrm{X}$ & $X$ \\
\hline & Digit Symbol-Coding & & $X$ & & $\mathrm{X}$ & $\mathrm{X}$ \\
\hline \multirow[t]{3}{*}{ Episodic memory } & Rivermead Behavioural Memory Test & & $X$ & & $\mathrm{X}$ & $\mathrm{X}$ \\
\hline & $\begin{array}{l}\text { Rey Complex Figure Test-immediate and delayed recall } \\
\text { trials }\end{array}$ & & $\mathrm{X}$ & & $\mathrm{X}$ & $\mathrm{X}$ \\
\hline & Verbal Paired Associates & & $X$ & & $\mathrm{X}$ & $X$ \\
\hline Working memory & Digit span Forward and Backward conditions & & $X$ & & $\mathrm{X}$ & $\mathrm{X}$ \\
\hline Language & Boston Naming Test-short version & & $X$ & & $\mathrm{X}$ & $\mathrm{X}$ \\
\hline \multirow[t]{5}{*}{ Executive functioning } & Controlled Oral Word Association Test-FAS & & $X$ & & $\mathrm{X}$ & $\mathrm{X}$ \\
\hline & Category Fluency Test: animals and occupations & & $X$ & & $\mathrm{X}$ & $\mathrm{X}$ \\
\hline & Stroop Colour Word Test-card 3 & & $\mathrm{X}$ & & $\mathrm{X}$ & $\mathrm{X}$ \\
\hline & Rule Shift Cards & & $\mathrm{X}$ & & $\mathrm{X}$ & $\mathrm{X}$ \\
\hline & Zoo Map & & $\mathrm{X}$ & & $\mathrm{X}$ & $\mathrm{X}$ \\
\hline Fine motor dexterity & Purdue Pegboard & & $\mathrm{X}$ & & $\mathrm{X}$ & $\mathrm{X}$ \\
\hline \multicolumn{7}{|l|}{ Secondary outcomes } \\
\hline \multirow[t]{2}{*}{ Quality of Life } & WHO Quality of Life Questionnaire-short form ${ }^{22}$ & & $\mathrm{X}$ & & $\mathrm{X}$ & $X$ \\
\hline & WHO Quality of Life Questionnaire-Old module ${ }^{23}$ & & $\mathrm{X}$ & & $\mathrm{X}$ & $\mathrm{X}$ \\
\hline \multicolumn{7}{|l|}{ ADL functioning } \\
\hline Basic ADL & Barthel index 25 & $\mathrm{P}$ & $X$ & $\mathrm{X}$ & $\mathrm{X}$ & $\mathrm{X}$ \\
\hline Instrumental ADL & Frenchay Activities Index ${ }^{26}$ & & $x$ & $\mathrm{X}$ & $\mathrm{X}$ & $x$ \\
\hline Subjective stroke recovery & Item 9 of Stroke Impact Scale ${ }^{24}$ & & $\mathrm{P}$ & & $\mathrm{P}$ & $\mathrm{P}$ \\
\hline
\end{tabular}

asked to participate in the study. The rest of the procedure is the same as that for the patient group.

The assessments are administered in a standardised way by trained neuropsychologists and take place at the participating hospitals or, when this is not possible, at the participant's home or residence (eg, rehabilitation centre).

\section{Measures}

Tables 1 and 2 give an overview of the variables assessed and instruments used at each time point.

\section{Outcomes}

Primary outcomes of the COMPAS study are SCC and OCP. To measure SCC, two instruments are used, namely: the Dutch version of the Cognitive Failures Questionnaire $(\mathrm{CFQ})^{16}{ }^{17}$ and the Checklist for Cognitive and Emotional consequences following stroke (CLCE-24). ${ }^{5}$ The CFQ focuses on SCCc and asks subjects to rate 25 items on the frequency of cognitive slips and errors in daily life on a 5-point Likert scale ranging from 0 (never) to 4 (very often). SCCw is evaluated by four additional general questions regarding the subjective increase of complaints over time, the degree to which these hinder daily life, are annoying and are a source of concern. Each of these extra items is rated on a scale ranging from 1 (not at all) to 5 (extremely).

The CLCE-24 is a structured clinical interview developed to evaluate SCCC and SCCw among stroke survivors. $^{5}$ It consists of 13 items concerning cognitive complaints and 9 items addressing emotional and behavioural complaints. Each item is scored as 0 (not present), 1 (doubtful), 2 (present, but not affecting daily life) or 3 (present and negatively affecting daily life).

OCP are evaluated using an extensive neuropsychological assessment covering multiple cognitive domains and containing traditional (eg, Rey Complex Figure Test $^{18}$ ) and more ecologically valid tests (eg, Rivermead Behavioural Memory Test ${ }^{19}$ ). See table 1 for an overview of all OCP tests used. In Spreen and Straus $^{20}$ and Lezak et $a l^{21}$ a detailed description of each of the instruments we use is given.

Secondary outcomes include QoL, ADL functioning and subjective stroke recovery. Generic QoL is evaluated using the short version of the self-report WHO Quality of Life Questionnaire (WHOQOL-Bref) ${ }^{22}$ (26 items) and, because we expect the majority of our population 
Table 2 Possible determinants in the COMPAS study

\begin{tabular}{|c|c|c|c|c|c|c|}
\hline & Variable/instrument & TO & T1 & T2 & T3 & T4 \\
\hline Demographic variables & $\begin{array}{l}\text { Age, gender, education, marital status, living situation, residence, } \\
\text { employment status, hand preference }\end{array}$ & $\mathrm{P}$ & $X$ & $\mathrm{X}$ & $\mathrm{X}$ & $\mathrm{X}$ \\
\hline \multicolumn{7}{|l|}{ Clinical characteristics } \\
\hline Stroke-specific & $\begin{array}{l}\text { Lifetime history of stroke, type, side, classification according to the } \\
\text { Oxford Community Stroke Project, }{ }^{27} \text { severity within } 24 \mathrm{~h} \text { after admission } \\
\text { using the National Institutes of Health Stroke Scale, }{ }^{28} \text { treatment, } \\
\text { poststroke complications, length of hospital stay, discharge destination }\end{array}$ & $\mathrm{P}$ & & & & \\
\hline General & $\begin{array}{l}\text { Vascular risk factors, comorbidity (Cumulative Illness Rating } \text { Scale }^{29} \text { ), } \\
\text { (re-) admissions to hospital, medication use, current participation in } \\
\text { rehabilitation therapy }\end{array}$ & & $\mathrm{X}$ & $\mathrm{X}$ & $\mathrm{X}$ & $\mathrm{X}$ \\
\hline Health status & 12-Item Short Form Health Survey ${ }^{30}$ & & $X$ & & $\mathrm{X}$ & $\mathrm{X}$ \\
\hline \multicolumn{7}{|l|}{ Premorbid status } \\
\hline Cognitive decline & $\begin{array}{l}\text { Informant Questionnaire on Cognitive Decline in the Elderly-short } \\
\text { form }^{15}\end{array}$ & $\mathrm{P}$ & $\mathrm{C}$ & & & \\
\hline IQ estimation & Dutch version National Adult Reading Test ${ }^{31}$ & & $\mathrm{X}$ & & & \\
\hline Cognitive complaints & $\begin{array}{l}\text { Self-made item: "in the previous months (before your stroke), have you } \\
\text { experienced cognitive complaints?" }\end{array}$ & $\mathrm{P}$ & C & & & \\
\hline $\begin{array}{l}\text { Depressive } \\
\text { complaints }\end{array}$ & $\begin{array}{l}\text { Self-made item: "in the previous months (before your stroke), have you } \\
\text { experienced depressive complaints?" }\end{array}$ & $\mathrm{P}$ & $\mathrm{C}$ & & & \\
\hline Anxiety complaints & $\begin{array}{l}\text { Self-made item: "in the previous months (before your stroke), have you } \\
\text { experienced anxiety complaints?" }\end{array}$ & $\mathrm{P}$ & $\mathrm{C}$ & & & \\
\hline \multicolumn{7}{|c|}{ Current comorbid complaints } \\
\hline $\begin{array}{l}\text { Depressive } \\
\text { complaints }\end{array}$ & Hospital Anxiety and Depression Scale-subscale depression ${ }^{32}$ & & $\mathrm{X}$ & $\mathrm{X}$ & $\mathrm{X}$ & $\mathrm{X}$ \\
\hline Anxiety complaints & Hospital Anxiety and Depression Scale-subscale anxiety ${ }^{32}$ & & $\mathrm{X}$ & $\mathrm{X}$ & $\mathrm{X}$ & $\mathrm{X}$ \\
\hline Fatigue & Fatigue Assessment Scale ${ }^{33}$ & & $\mathrm{X}$ & $\mathrm{X}$ & $\mathrm{X}$ & $\mathrm{X}$ \\
\hline Stress & Perceived Stress Scale, 4-item version ${ }^{34}$ & & $\mathrm{X}$ & & $\mathrm{X}$ & $\mathrm{X}$ \\
\hline Personal factors & & & $\mathrm{X}$ & & $\mathrm{X}$ & $\mathrm{X}$ \\
\hline Coping style & Utrecht Coping List-15-item version ${ }^{35}$ & & $X$ & & & \\
\hline $\begin{array}{l}\text { Personality trait- } \\
\text { neuroticism }\end{array}$ & $\begin{array}{l}\text { Eysenck Personality Questionnaire Revised Short Scale-subscale } \\
\text { neuroticism }{ }^{36}\end{array}$ & & $\mathrm{X}$ & & & \\
\hline $\begin{array}{l}\text { Personality trait- } \\
\text { extraversion }\end{array}$ & $\begin{array}{l}\text { Eysenck Personality Questionnaire Revised Short Scale-Extraversion } \\
\text { subscale }^{36}\end{array}$ & & $x$ & & & \\
\hline Type D & Type D scale- $14^{37}$ & & $\mathrm{X}$ & & & \\
\hline Participants' & Cognitive Failures Questionnaire completed by proxy & & $\mathrm{X}$ & $\mathrm{X}$ & $\mathrm{X}$ & $\mathrm{X}$ \\
\hline awareness of SCC & $\begin{array}{l}\text { Checklist for Cognitive and Emotional Consequences completed by } \\
\text { proxy }\end{array}$ & & $\mathrm{X}$ & $\mathrm{X}$ & $\mathrm{X}$ & $\mathrm{x}$ \\
\hline Life events & $\begin{array}{l}\text { Self-made item concerning the presence and impact of a positive or } \\
\text { negative life event: "Last year, did something happen in your life which } \\
\text { had a major impact on you? This may be something either pleasant or } \\
\text { sad" }\end{array}$ & & $\mathrm{X}$ & & $\mathrm{X}$ & $\mathrm{X}$ \\
\hline
\end{tabular}

to be elderly ( $>60$ years), the additional OLD module (WHOQOL-OLD) ${ }^{23}$ comprising 24 items. While the first covers overall well-being on the domains 'physical', 'psychological', social relationships' and 'environment', the OLD module evaluates aspects of life which are specific to the elderly, including: 'intimacy', 'sensory abilities', 'autonomy', 'activities in the past, present and future', 'social participation' and 'dying'.

Subjective recovery after stroke is determined by a single item from the Stroke Impact Scale, ${ }^{24}$ in which patients are asked to indicate on a scale ranging from 0 ('no recovery') to 100 ('full recovery') how much they feel they have recovered from their stroke.
ADL functioning is assessed in basic activities, including self-care and mobility, using the Barthel Index ${ }^{25}$ (10 items) and more complex activities like housekeeping, hobbies and employment, using the Frenchay Activities Index $^{26}$ (15 items).

All instruments chosen are frequently used (inter) nationally in research and daily clinical practice dealing with stroke patients.

\section{Possible determinants}

Depending on the specific outcome considered, SCC, OCP, QoL, subjective recovery and ADL functioning are either dependent or independent variables. A wide range 
of possible determinants are additionally taken into account, based on what is currently known from the literature on SCC in the general and the stroke population. These include: demographic variables, clinical characteristics (those related to stroke included) and health status; premorbid status (ie, cognitive decline, IQ cognitive and emotional complaints); comorbid complaints about mood (ie, anxiety and depression), fatigue and stress; personal factors (ie, coping style, personality traits and SCC awareness), and the occurrence and impact of positive and/or negative live events. See table 2 for the specific variables assessed and instruments used.

\section{Planned statistical analyses}

Cross-sectional analyses will be used to evaluate group differences at each of the individual time points (T1-T4) and include: $\chi^{2}$ test for categorical variables, the Mann-Whitney $\mathrm{U}$ test for ordinal data, and the Student t test or (multivariate) analysis of variances ((M)ANOVA) for continuous dependent variables. Furthermore, differences across the time points will be analysed using multilevel analysis, which allows inclusion of all available data (ie, also those from participants with partly missing values).

The course of SCC over time (T1-T4) will subsequently be evaluated using latent class growth analysis. We will explore whether groups with different trajectories of SCC over time can be distinguished and, if so, what their characteristics are.

The predictive value of the determinants for the primary and secondary outcome measures (ie, SCC, OCP, QoL, subjective recovery and ADL functioning) at T3 and $\mathrm{T} 4$ will be determined using multivariate regression analysis. Potential predictors are defined as variables with at least a marginally significant association $(p<0.10)$ with the outcome. Only these variables will be included in the subsequent regression analyses to determine the most important predictors. In general, effects with a two-tailed $\mathrm{p}<0.05$ are considered statistically significant.

\section{Sample size and power calculation}

The sample size needed in the COMPAS study is calculated using the method for multilevel analysis according to Twisk. ${ }^{38}$ Based on a high intraindividual correlation across the different time points $(\mathrm{r}=0.70)$, an $\alpha$ level of 0.05 and power of 0.80 , there are 180 participants per group needed to be able to detect a small difference (at least $0.2 \mathrm{SD}$ ) between the groups. We expect about $40 \%$ drop-outs during the 2-year follow-up period due to mortality, comorbidity or refusal to continue participation. Therefore, we aim to include 300 participants at baseline in each group in order to end up with the 180/ group needed.

\section{ETHICS AND DISSEMINATION Ethical considerations}

The COMPAS study is conducted in accordance with the 'Helsinki Declaration' (Seoul revision, 2008) and the 'medical Research Involving Human Subjects Act' (WMO). The study is non-invasive, imposes no risk on participants, and its protocol has been approved by the medical ethical committees of all participating hospitals (ie, St. Elisabeth and TweeSteden Hospitals in Tilburg, and the Maxima Medical Centre in Veldhoven), and has been registered by the Central committee on Research Involving Human Subjects (number NL31208.008.10). Furthermore, written informed consent is obtained from all participants.

\section{Dissemination}

The results obtained will be disseminated to the scientific, medical and general public by publication in national and international peer-reviewed journals, as well as by presentations in conferences and meetings with clinicians dealing with patients with stroke.

\section{DISCUSSION}

The COMPAS study is the first in which poststroke SCC are systematically evaluated over time, while a wide range of subjective and objective variables in patients and controls is taken into account. While numerous studies have measured poststroke OCP, only a few have also evaluated the patients' SCC. Also, while these symptoms are found to be common among patients with stroke, knowledge about their risk factors, their course over time, differences with the non-stroke population and their predictive value for future functioning is practically non-existent.

Strong elements of the COMPAS study are its prospective design with multiple assessments during the first two years after stroke, and the extensive evaluations of subjective and objective variables, which, based on the current literature, are potentially relevant to SCC after stroke. This gives us the opportunity to determine a detailed risk profile for experiencing poststroke SCC. Furthermore, the instruments chosen are widely accepted and frequently used in daily clinical practice dealing with patients with stroke. Traditional neuropsychological and more ecologically valid tests (eg, the Rivermead Behavioural Memory Test) are used to evaluate OCP, making it possible to determine whether the ecological validity of tests affects the association between SCC and OCP. Also, a healthy control group is assessed at the same time points as the patients and will be used as a reference group. This enables us to distinguish poststroke SCC in their prevalence, profile and time course from, for example, factors which are associated with ageing. A potential limitation of the study is that the most seriously affected patients with stroke are unable to participate, thereby reducing the possibility to generalise the results to the stroke population as a whole. However, our study differs from those carried out until now in this field in that we include a broad selection of patients with stroke, not only first-ever strokes or patients discharged home. 
In conclusion, we feel that the COMPAS study has the potential to contribute to knowledge on poststroke SCC. Owing to the ageing population and healthcare improvements, the number of stroke survivors who will have to deal with poststroke impairment will increase in the future, and the social and economic burdens will rise accordingly. ${ }^{39} 40$ Clinicians are frequently confronted with patients having SCC after their stroke, but the meaning and relevance of these SCG have yet to be determined. We aim to elucidate the possible risk factors, prognosis and the predictive value of poststroke SCC. This information can subsequently be applied by clinicians in daily practice in order to more accurately inform patients and their proxies and to treat SCC. Our data may also prove useful in the future development of patient-tailored intervention programmes to ultimately improve individual stroke patient-centred care, which is the ultimate aim of the COMPAS study.

\section{Author affiliations}

${ }^{1}$ Department of Cognitive Neuropsychology, Centre of Research on Psychology in Somatic diseases (CoRPS), Tilburg University, Tilburg, The Netherlands

${ }^{2}$ Department of Neurology, St Elisabeth Hospital, Tilburg, The Netherlands

Contributors RM, PK and MR conceptualised the study. MS and RM contributed to the procurement of funding. MR, RM and PK developed procedures for implementing the protocol. All authors contributed to and have checked the final manuscript.

Funding This study is financially supported by the Centre of Research on Psychology in Somatic diseases (CoRPS) from Tilburg University, The Netherlands.

Competing interests None.

Ethics approval Medical Ethics Committee St Elisabeth Hospital, TweeSteden Hospital in Tilburg, and Maxima Medical Centre in Veldhoven.

Provenance and peer review Not commissioned; internally peer reviewed.

Open Access This is an Open Access article distributed in accordance with the Creative Commons Attribution Non Commercial (CC BY-NC 3.0) license, which permits others to distribute, remix, adapt, build upon this work noncommercially, and license their derivative works on different terms, provided the original work is properly cited and the use is non-commercial. See: http:// creativecommons.org/licenses/by-nc/3.0/

\section{REFERENCES}

1. Lamb F, Anderson J, Saling M, et al. Predictors of subjective cognitive complaint in post-acute older adult stroke patients. Arch Phys Med Rehabil 2013;94:1747-52.

2. Winkens I, Van Heugten CM, Fasotti L, et al. Reliability and validity of two new instruments for measuring aspects of mental slowness in the daily lives of stroke patients. Neuropsychol Rehabil 2009;19:64-85.

3. van Rijsbergen MW, Mark RE, de Kort PL, et al. Subjective cognitive complaints after stroke: a systematic review. J Stroke Cerebrovasc Dis. Published Online First: 22 June 2013. doi:10.1016/j.jstrokecerebrovasdis. 2013.05.003

4. Duits A, Munnecom T, van Heugten $\mathrm{C}$, et al. Cognitive complaints in the early phase after stroke are not indicative of cognitive impairment. J Neurol Neurosurg Psychiatry 2008;79:143-6.

5. van Heugten $\mathrm{C}$, Rasquin S, Winkens I, et al. Checklist for cognitive and emotional consequences following stroke (CLCE-24): development, usability and quality of the self-report version. Clin Neurol Neurosurg 2007;109:257-62.

6. Wilz G, Barskova T. Predictors of psychological and somatic components of poststroke depression: a longitudinal study. Top Stroke Rehabil 2007;14:25-40.
7. Jonker C, Geerlings MI, Schmand B. Are memory complaints predictive for dementia? A review of clinical and population-based studies. Int J Geriatr Psychiatry 2000;15:983-91.

8. Reid LM, Maclullich AM. Subjective memory complaints and cognitive impairment in older people. Dement Geriatr Cogn Disord 2006;22:471-85.

9. Comijs HC, Deeg DJ, Dik MG, et al. Memory complaints; the association with psycho-affective and health problems and the role of personality characteristics. A 6-year follow-up study. J Affect Disord 2002;72:157-65.

10. Jorm AF, Butterworth $\mathrm{P}$, Anstey $\mathrm{KJ}$, et al. Memory complaints in a community sample aged 60-64 years: associations with cognitive functioning, psychiatric symptoms, medical conditions, APOE genotype, hippocampus and amygdala volumes, and white-matter hyperintensities. Psychol Med 2004;34:1495-506.

11. Paradise MB, Glozier NS, Naismith SL, et al. Subjective memory complaints, vascular risk factors and psychological distress in the middle-aged: a cross-sectional study. BMC Psychiatry 2011; 11:108.

12. Mol M, Carpay M, Ramakers I, et al. The effect of perceived forgetfulness on quality of life in older adults; a qualitative review. Int J Geriatr Psychiatry 2007;22:393-400.

13. Berg A, Palomaki $\mathrm{H}$, Lonnqvist J, et al. Depression among caregivers of stroke survivors. Stroke 2005;36:639-43.

14. van Exel $\mathrm{NJ}$, Koopmanschap MA, van den Berg B, et al. Burden of informal caregiving for stroke patients. Identification of caregivers at risk of adverse health effects. Cerebrovasc Dis 2005;19:11-17.

15. de Jonghe JF, Schmand B, Ooms ME, et al. [Abbreviated form of the Informant Questionnaire on cognitive decline in the elderly]. Tijdschr Gerontol Geriatr 1997;28:224-9.

16. Broadbent DE, Cooper PF, FitzGerald $\mathrm{P}$, et al. The Cognitive Failures Questionnaire (CFQ) and its correlates. Br J Clin Psychol 1982;21(Pt 1):1-16.

17. Ponds $\mathrm{R}$, van Boxtel M, Jolles J. [The 'Cognitive Failure Questionnaire' as a measure of subjective cognitive functioning]. Tijdschrift voor neuropsychologie-diagnostiek, behandeling en onderzoek 2006;1:37-45.

18. Osterrieth PA. [The test of copying a complex figure: a contribution to the study of perception and memory]. Archives de Psychologie 1944;30:206-353.

19. Wilson B, Cockburn J, Baddeley A, et al. The development and validation of a test battery for detecting and monitoring everyday memory problems. J Clin Exp Neuropsychol 1989;11:855-70.

20. Spreen O, Strauss E. A compendium of neuropsychological tests administration, norms and commentary. 2nd edn. New York: Oxford University Press, 1998.

21. Lezak MD, Howieson DB, Loring DW, et al. Neuropsychological assessment. 4th edn. New York: Oxford, 2004.

22. Skevington SM, Lotfy M, O'Connell KA. The World Health Organization's WHOQOL-BREF quality of life assessment: psychometric properties and results of the international field trial. A report from the WHOQOL Group. Qual Life Res 2004; 13:299-310.

23. Power M, Quinn K, Schmidt S. Development of the WHOQOL-old module. Qual Life Res 2005;14:2197-214.

24. Williams LS, Weinberger M, Harris LE, et al. Development of a stroke-specific quality of life scale. Stroke 1999;30:1362-9.

25. de Haan R, Limburg M, Schuling J, et al. [Clinimetric evaluation of the Barthel Index, a measure of limitations in activities of daily living]. Ned Tijdschr Geneeskd 1993;137:917-21.

26. Holbrook M, Skilbeck CE. An activities index for use with stroke patients. Age Ageing 1983;12:166-70.

27. Bamford J, Sandercock P, Dennis $M$, et al. Classification and natura history of clinically identifiable subtypes of cerebral infarction. Lancet 1991;337:1521-6.

28. Brott T, Adams HP Jr., Olinger CP, et al. Measurements of acute cerebral infarction: a clinical examination scale. Stroke 1989;20:864-70.

29. Linn BS, Linn MW, Gurel L. Cumulative illness rating scale. J Am Geriatr Soc 1968;16:622-6.

30. Ware J Jr., Kosinski M, Keller SD. A 12-Item Short-Form Health Survey: construction of scales and preliminary tests of reliability and validity. Med Care 1996;34:220-33.

31. Schmand B, Bakker D, Saan R, et al. [The Dutch Adult Reading Test: a measure of premorbid intelligence]. Tijdsch Gerontol Geria 1991;22:15-19.

32. Zigmond AS, Snaith RP. The hospital anxiety and depression scale. Acta Psychiatr Scand 1983;67:361-70.

33. Michielsen HJ, De Vries J, Van Heck GL. Psychometric qualities of a brief self-rated fatigue measure: The Fatigue Assessment Scale. J Psychosom Res 2003;54:345-52. 
34. Cohen S, Kamarck T, Mermelstein R. A global measure of perceived stress. J Health Soc Behav 1983;24:385-96.

35. Sanderman R, Ormel J. [The Utrecht Coping List (UCL): validity and reliability]. Gedrag Gezond 1992;20:32-7.

36. Sanderman R, Arrindell WA, Ranchor A, et al. [Measurement of personality traits using the Eysenck Personality Questionnaire: a manual]. Groningen: Noordelijk Centrum voor

Gezondheidsvraagstukken, Rijksuniversiteit Groningen, 1995.
37. Denollet J. DS14: standard assessment of negative affectivity, social inhibition, and Type D personality. Psychosom Med 2005;67:89-97.

38. Twisk JWR. Applied longitudinal data analysis for epidemiology. New York: Cambridge University Press, 2003.

39. Donnan GA, Fisher M, Macleod M, et al. Stroke. Lancet 2008;371:1612-23.

40. Strong K, Mathers $\mathrm{C}$, Bonita R. Preventing stroke: saving lives around the world. Lancet Neurol 2007;6:182-7. 